\title{
Profile of fungal keratitis in a Sub-Himalayan territory of north India
}

\author{
Anil Kumar Verma', Anuradha Sood², Anil Chauhan', Rajeev Tuli', \\ Subhash Chand Jaryal ${ }^{2}$ \\ 'Department of Ophthalmology, Dr. Rajendra Prasad Government Medical \\ College Kangra at Tanda, Himachal Pradesh, India; ${ }^{2}$ Department of Microbiology, \\ Dr. Rajendra Prasad Government Medical College Kangra at Tanda, Himachal \\ Pradesh, India
}

\begin{abstract}
Aim: To study the microbiological and epidemiological profile of patients with suppurative corneal ulcer presenting in a rural referral center situated in a Sub-Himalayan territory of north India. The study was conducted to evaluate the epidemiology and frequency of mycotic keratitis among the patients of suppurative corneal ulcer and to identify various fungal species as etiological agents.

Methods: Corneal scrapings from 56 patients of suppurative corneal ulcers were subjected to direct microscopy and culture.

Results: Of the 56 cases of suppurative corneal ulcer investigated, fungal etiology was identified in 18 (32\%) cases. Most of the patients (82.1\%) worked in agriculture. Trivial trauma with vegetative matter was the most common predisposing factor. Fusarium and Acremonium species were the most common fungi isolated, followed by Aspergillus. Four cases of rare mycotic keratitis caused by Paecilomyces lilacinus, Scedosporium apiospermum, Monilia sitophila, and Ulocladium species were detected. Four cases were smear positive (10\% $\mathrm{KOH}$ wet mount) but culture negative. Analysis of $\mathrm{KOH}$ wet mount was done using culture as gold standard. The sensitivity and specificity of $\mathrm{KOH}$ wet mount was $71.43 \%$ and $90.48 \%$, respectively.
\end{abstract}

Conclusion: Direct microscopy and culture has a greater diagnostic value in the management of suppurative corneal ulcer. The authors have observed changes in the pattern of organisms identified as cause of fungal keratitis in the region. Rare species of fungi may also be detected if corneal scrapings are collected for direct microscopy and culture from all the cases of suppurative corneal ulcers greater than $2 \mathrm{~mm}$.

Keywords: corneal ulcer, fungal keratitis, north India, rare fungi, Sub-Himalayan

Correspondence: Dr. Anil Kumar Verma (MS Ophthalmology), Department of Ophthalmology, Dr. Rajendra Prasad Government Medical College Kangra at Tanda, Kangra, Himachal Pradesh 17600, India.

E-mail: anilkumarverma2971@gmail.com 


\section{Introduction}

Fungal infections of the eye are recognized as an important cause of morbidity and blindness worldwide. The prevalence of individual pathogens largely depends on geographical and climatic factors. Due to a large agrarian population and environmental factors, mycotic keratitis is common in India. Direct microscopy, a simple and widely available laboratory technique, is of greater diagnostic value in the management of suppurative corneal ulcers. The diversity of clinical presentations observed in each case and also new emerging cases each year pose a diagnostic and therapeutic challenge to the ophthalmologist. It shows greater morbidity than bacterial keratitis because of delayed diagnosis and available drugs that may not be effective. Newer and rare fungi are being reported all over the globe. More than 70 genera of filamentous fungi and yeasts have been identified as the etiological agents of mycotic keratitis. ${ }^{1-4}$

This study was undertaken to evaluate the frequency of positive fungal cultures in suppurative corneal ulcers and of various fungal species identified as etiological agents in the patients attending a rural referral centre in a Sub-Himalayan territory of north India.

\section{Material and methods}

A total of 56 cases of suppurative corneal ulcer were studied. These patients presented during a 2-year period. Patients with suspected or confirmed viral keratitis, healing corneal ulcers, neuroparalytic keratitis, interstitial keratitis, ulcers associated with autoimmune conditions, cornea at risk of perforation, small ulcers (less than $2 \mathrm{~mm}$ size), and patients who refused to participate were excluded from the study. Patients were examined using standardized protocols and proforma. A detailed ocular examination was performed. To determine the causative organism, corneal scrapings were taken under magnification of slit-lamp biomicroscope for direct microcopy and culture. After topical anesthesia with xylocaine $4 \%$, the base and the leading edge of the ulcers were scraped from periphery to centre with the blunt edge of no. 15 disposable blades. The material was smeared on two slides, i.e., wet mount $\mathrm{KOH} 10 \%$ and Gram staining. It was smeared thinly with a marked area on the glass slides for Gram staining. For KOH 10\% preparation, scrapings were placed within a marked area on glass slide and then covered with one drop of $\mathrm{KOH} 10 \%$, followed by placement of cover slip. The material was then inoculated onto blood agar and Sabouraud's dextrose agar (SDA) with and without antibiotic, followed by subculture on solid medium. Two sets of SDA with and without antibiotic were maintained at $25^{\circ} \mathrm{C}$ and $37^{\circ} \mathrm{C}$ separately over a period of 4 weeks. The inoculated SDA was examined daily and discarded at 3 weeks if no growth was seen. If fungal hyphae were seen on $\mathrm{KOH}$ smear, but failed to grow in culture, the causative organism was reported as fungi. 
A treatment protocol was followed. After taking the corneal scrapings for direct microcopy and culture, the patient was put on empirical treatment consisting of fortified cefazoline $5 \%$, fortified tobramycin $1.3 \%$, atropine eye drops $1 \%$, tablet acetazolamide $15 \mathrm{mg} / \mathrm{kg} /$ day. If $\mathrm{KOH}$ wet mount was positive, natamycin $5 \%$ drops were added to the treatment regime. Oral dose of itraconazole $100 \mathrm{mg}$ was given BID in non-responding cases.

\section{Results}

A total of 56 patients met the inclusion criteria of this study. Thirty (53.6\%) patients were male and $26(46.4 \%)$ were female. All cases lived in rural areas. The most common affected age group was 41-60 years (37.6\%). The mean age was 52.7 years (range $22-95$ years). Forty-one cases (73.2\%) worked in agriculture (Table 1 ). Corneal injury (44 cases, 78.6\%) was the most common predisposing factor (Table 2). Trivial trauma with vegetative matter (35 cases, $62.5 \%$ ) was the most common mode of injury (Table 3 ). The majority of patients (38 cases, $67.9 \%$ ) had consulted health care providers of some kind before presenting to our referral rural center and had used topical medications such as antibiotics, antifungals, and steroids, or traditional medicines such as ayurvedic eye drops, plant juice, etc.

Table 1. Occupation distribution of subjects

\begin{tabular}{|l|l|l|l|l|l|l|}
\hline Occupation & Agriculture & Laborer & Mechanic & Driver & Other & Total \\
\hline Cases (\%) & $41(73.2 \%)$ & $6(10.7 \%)$ & $3(5.3 \%)$ & $2(3.7 \%)$ & $4(7.1 \%)$ & 56 \\
\hline
\end{tabular}

Table 2. Distribution of subjects according to their predisposing factors

\begin{tabular}{|l|l|}
\hline Ocular factors & No. of cases \\
\hline Corneal injury & 44 \\
\hline Lagophthalmos* $^{*}$ & 3 \\
\hline Chronic dacryocystitis* $^{*}$ & 3 \\
\hline Trichiasis & 2 \\
\hline Use of topical steroids & 5 \\
\hline Ectropion & 1 \\
\hline Entropion & 1 \\
\hline Systemic factors & \\
\hline Diabetes mellitus* & 4 \\
\hline None & 5 \\
\hline
\end{tabular}

*Some patients had more than one predisposing factor 
Table 3. Distribution of subjects according to mode of injury $(n=44)$

\begin{tabular}{|l|l|l|l|l|l|l|}
\hline Traumatic agents & $\begin{array}{l}\text { Vegetative } \\
\text { matter }\end{array}$ & Dust & Stone & Metal & Toothbrush & Total \\
\hline No. of cases & 35 & 3 & 3 & 2 & 1 & 44 \\
\hline
\end{tabular}

Table 4. Distribution of subjects according to fungal isolates detected from culture $(n=14)$

\begin{tabular}{|l|l|l|l|}
\hline Fungi & Pure isolates & $\begin{array}{l}\text { Mixed with } \\
\text { bacteria }\end{array}$ & Total \\
\hline Acremonium & 4 & & 4 \\
\hline Aspergillus flavus & 1 & 1 & 2 \\
\hline Fusarium & 4 & & 4 \\
\hline Peacilomyces lilacinus & 1 & & 1 \\
\hline Monilia sitophila & 1 & & 1 \\
\hline Scedosporium apiospermum & 1 & & 1 \\
\hline Ulocladium & 1 & & 1 \\
\hline Total & $\mathbf{1 3}$ & $\mathbf{1}$ & $\mathbf{1 4}$ \\
\hline
\end{tabular}

Most patients were from low socioeconomic status (41 cases, 73.2\%). A significant increase in the number of cases was observed during the harvesting seasons between March and April (19 cases, 33.9\%) and November and December (14 cases, 25\%). Thirty-one cases had an associated hypopyon. Site of the ulcer was central in 38 cases and paracentral in 18 cases. Forty-nine cases $(87.5 \%)$ were treated as inpatients and seven cases (12.5\%) were treated as outpatients. Mean duration of inpatient treatment was 13.46 days (range 3-29 days).

Of 56 cases of suppurative keratitis, a fungal etiology was identified in 18 cases. Of these, 11 were male and 7 were female. All cases were of unilateral mycotic keratitis. Among 18 cases of fungal keratitis 10 cases were both culture- and smear-positive, 4 cases were culture-positive and smear-negative, and 4 cases were smear-positive and culture-negative. Among 14 culture-positive cases, the most frequent agents isolated were Fusarium (4 cases) and Acremonium species (4 cases), followed by Aspergillus flavus (2 cases). One case each of Paecilomyces lilacinus, Scedosporium apiospermum, Monilia sitophila, and Ulocladium species were detected (Table 4). Analysis of $\mathrm{KOH}$ wet mount was done using culture as gold standard. The sensitivity and specificity of $\mathrm{KOH}$ wet mount was $71.43 \%$ and $90.48 \%$, respectively. The positive predictive value and negative predictive value of $\mathrm{KOH}$ wet mount was $71.42 \%$ and $90.47 \%$, respectively (Table 5 ). 
Table 5. $\mathrm{KOH}$ wet mount using culture as gold standard $(n=56)$

\begin{tabular}{|l|l|l|}
\hline \multicolumn{2}{|l|}{ KOH Wet mount culture } & \multirow{2}{*}{ Total } \\
\cline { 1 - 2 } Positive & Negative & \\
\hline 10 & 4 & $\mathbf{1 4}$ \\
\hline 4 & 38 & $\mathbf{4 2}$ \\
\hline $\mathbf{1 4}$ & $\mathbf{4 2}$ & $\mathbf{5 6}$ \\
\hline
\end{tabular}

Sensitivity: $71.43 \%$

Specificity: $90.48 \%$

Positive predictive value: $71.42 \%$

Negative predictive value: $90.47 \%$

\section{Discussion}

Corneal infections are known to be the second most significant cause of monocular blindness rated after unoperated cataract in some developing nations in particular and in the tropics in general. Limited technical and infrastructural capability for diagnosis and research as well as inaccessible treatment pose considerable challenges in the management of fungal keratitis in developing countries. The etiological and epidemiological patterns of corneal ulceration have been found to vary with the patient population, health of the cornea, geographic location and climate, and time. Hence, an understanding of the epidemiological and etiological features as well as the risk factors that occur in a specific region are important in rapid recognition, timely institution of therapy, optimal management, and prevention of this disease. In order to start specific therapy, it is necessary to conduct meticulous laboratory investigations which include microscopy and culture of corneal scrapings for identification of the microbial agent. ${ }^{5-8}$

In this Sub-Himalayan territory of north India, suppurative corneal ulcer continues to be a cause of concern; the predominance of agricultural activity is the principal causative factor. Most of these ulcers follow minor agricultural injuries. Corneal ulcers and ocular trauma are the commonest cause of corneal blindness, defined as "visual acuity less than 20/400 in one eye caused by corneal disease"..-11

In the present study, all patients (100\%) were from a rural background. We observed that illiterate individuals were more prone to corneal ulceration. Song et al. ${ }^{9}$ and Gupta et al. ${ }^{12}$ also observed that rural populations with low education levels, little or no access to health care, from lower socioeconomic status, with poor knowledge about proper eye care, and little awareness regarding preventive measures were more prone to develop suppurative corneal ulcers following trivial trauma. 


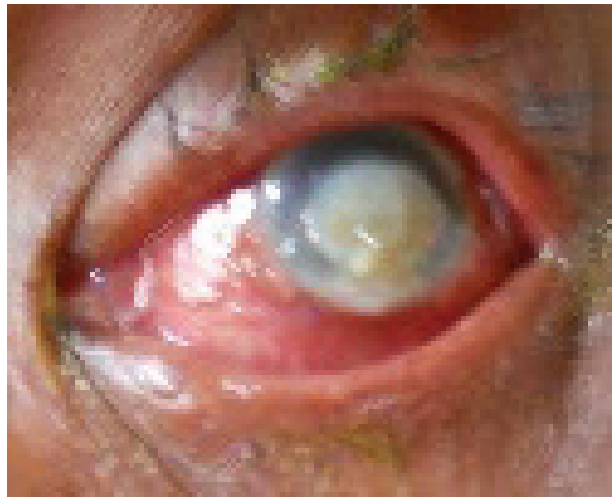

Fig. 1. Dry, leathery, and raised fungal corneal ulcer with thick hypopyon.

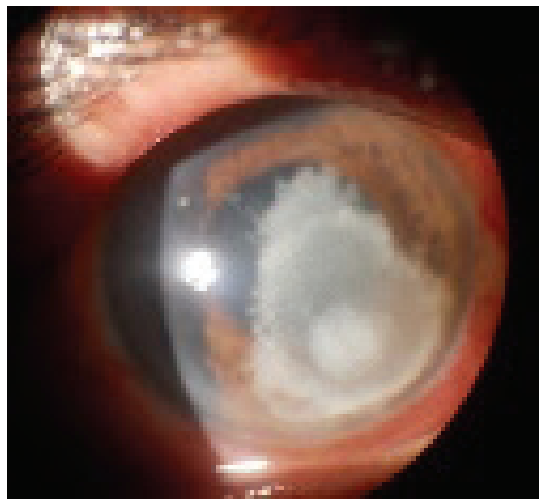

Fig. 2. Fungal corneal ulcer with irregular and feathery margins.

Most patients presented during the harvesting season, when agricultural activities are more intensive, due to which individuals are more prone to trivial corneal injuries with vegetative matter. Taylor et al. ${ }^{13}$ and Bharati et al. ${ }^{14}$ found a very high prevalence of fungal keratitis in rural India and China; furthermore, the incidence of keratitis was greater at the times of the year when agricultural work intensified.

It was observed that corneal injury was the most common predisposing factor causing suppurative keratitis. Vegetative matter was observed to be the most common traumatic agent. Titiyal ${ }^{15}$ and Srinivasan et al. ${ }^{16}$ also reported agricultural products as the main agents responsible for trauma.

Forty-five patients had consulted health care providers before presenting to our institute, having used some kind of topical medication including antibiotics, antifungals, ayurvedic eye drops, topical steroids, plant juice, seeds etc. Five patients had visited traditional healers before presentation. In their study of infectious keratitis in Delhi, Saha et al. found that 3.5\% patients gave history of use of topical herbal medicine before presentation. ${ }^{1}$ They further stated that the topical use of breast milk, castor oil, onion extract, flower extracts, honey, steam, and even chicken blood has been reported in south India.

Among 14 fungal culture-positive cases, Fusarium and Acremonium species were detected in four cases each, Aspergillus flavus was detected in two cases, and Scedosporium apiospermum, Monilia sitophila, Paecilomyces lilacinus, and Ulocladium were detected in one case each (Table 4). Analysis using culture as the gold standard revealed sensitivity and specificity of KOH wet mount as 71.43 $\%$ and $90.48 \%$, respectively. Ong et al. ${ }^{17}$ also observed the altered pattern of fungal keratitis at a London ophthalmic referral hospital. Ansari et al. ${ }^{18}$ found that although Fusarium and Aspergillus were most commonly associated with fungal keratitis, many other species such as Scedosporium apiospermum, Culvaria, and 


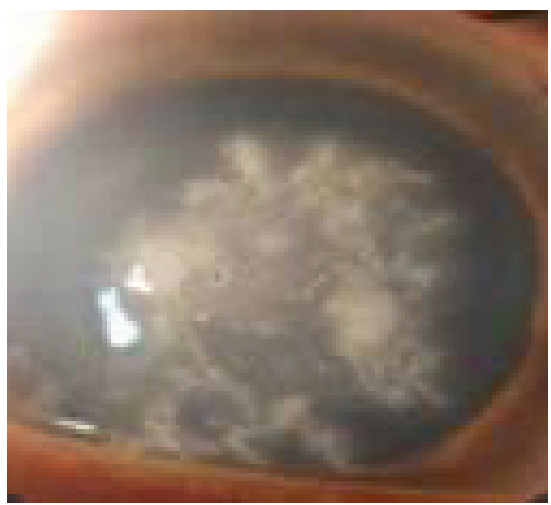

Fig. 3. Fungal corneal ulcer with pseudodendrites.

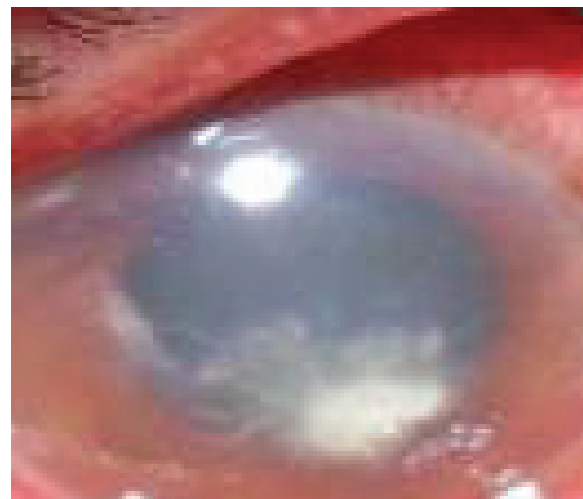

Fig. 4. Fungal corneal ulcer with satellite lesions.

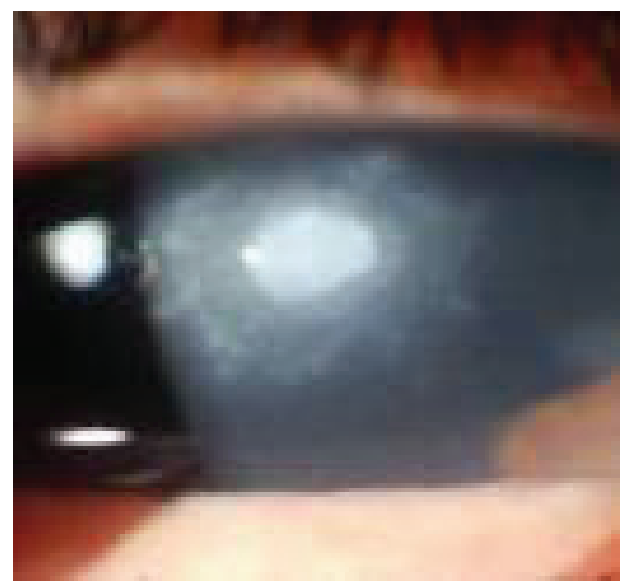

Fig. 5. Fungal corneal ulcer with Wessely immune ring.

Peacilomyces have also been robustly reported. It was observed that among 18 cases (14 culture-positive and 4 smear-positive but culture-negative) of fungal keratitis, 5 patients improved with topical natamycin alone, 9 patients improved with a combination of topical natamycin and oral itraconazole, and 3 patients were refractory to treatment and had the affected eye eviscerated. One subject with Scedosporium apiospermum keratitis was refractory to the above regime and responded only with topical voriconazole. On average, it took 6 weeks to 6 months to treat a case of fungal keratitis. Our observation did not match the study by Rajaram et al. ${ }^{19}$ which found that oral antifungal therapy did not add significant benefit to topical natamycin therapy in treating deep fungal keratitis. 
The diagnostic validity of traditional clinical features such as dry, raised, leathery ulcers with thick and immobile hypopyon (Fig. 1), pseudodendrites or feathery borders (Figs. 2 and 3), satellite lesions (Fig. 4), and Wessely immune rings (Fig. 5) has been challenged in recent years. However, we did observe these clinical features in our cases. That said, the utility of clinical diagnosis alone can be unreliable. ${ }^{18}$

Of 56 cases of suppurative keratitis, surgical procedures or other interventions were performed in 12 cases (25.8\%). Three cases with associated lagophthalmos were operated for lateral tarsorrhaphy, five cases which developed endophthalmitis due to perforation were eviscerated, two cases with descematocele were applied bandage contact lens, and two cases associated with chronic dacryocystitis were operated for dacryocystorrhinostomy. Extracorneal factors such as entropion, trichiasis, lagophthalmos, chronic dacryocystitis, and dry eye that directly or indirectly initiate or enhance keratitis should be treated meticulously. Despite advances in diagnosis and medical treatment of keratomycosis, $15-27 \%$ of patients require surgical intervention such as keratoplasty, enucleation, or evisceration due to either failed treatment or advanced disease at presentation..$^{20,21}$

We conclude that suppurative corneal ulcer is a major cause of preventable monocular blindness and educational strategies can reduce avoidable risk such as trauma, but treatment protocols are required to manage established disease. Direct microscopy and culture are of greater diagnostic value than traditional clinical features in the management of infective keratitis. The authors have observed changes in the pattern of organisms identified as cause of fungal keratitis. Rare mycotic keratitis may not be so uncommon if corneal scrapings are taken in all cases of suppurative corneal ulcers greater than $2 \mathrm{~mm}$ for direct microscopy and culture.

\section{Declarations}

\section{Ethics approval and consent to participate}

The study was approved by the Ethics Committee of Dr. Rajendra Prasad Government Medical College Kangra Tanda (India).

\section{Competing interests}

None to declare.

\section{Funding}

None to declare. 


\section{Acknowledgements}

The authors wish to acknowledge the invaluable support and help from all the staff members of the departments of Ophthalmology and Microbiology of Dr. Rajendra Prasad Government Medical College Kangra Tanda (India) for completing the study.

\section{References}

1. Saha R, Das S. Microbiological profile of infectious keratitis from Delhi. Indian J Med Res. 2006;123(2):159-164.

2. Chander J, Chakrabarti A, Sharma A, Saini JS. Mycotic keratitis: A five-year study from Chandigarh. Indian J Med Microbiol.1993;11:218-222.

3. Tuft SJ. Editorial: Suppurative keratitis. Br J Ophthalmol. 2003;87(2):127.

4. Mahapatra S, Sahu DK, Das G, Bhuyan DN, Behera S, Behera SK. Epidemiological and microbiological evaluation of microbial keratitis in Western Orissa; A two-year prospective study in a tertiary care centre. AIOS, 2009 Proceedings:304-306.

5. Tewari A, Sood N, Mahenrda M, Mehta D. Epidemiological and microbiological profile of infective keratitis in Ahmadabad. Indian J Ophthalmol. 2012;60(4):267-272.

6. Sharma S, Taneja M, Gupta R, et al. Comparison of clinical and microbiological profile in smear positive and smear negative cases of suspected microbial keratitis. Indian J Ophthalmol. 2007;50(1):21-25.

7. Haddad RS, El-Mollayess GM, Combination of intracameral and intrastromal voriconazole in the treatment of recalcitrant Acremonium fungal keratitis. Middle East Afr J Ophthalmol. 2012;19(2):265-268.

8. Guto JA, Bii CC, Denning DW. Estimated burden of fungal infections in Kenya. J Infect Dev Ctries. 2016;10(8):777-784.

9. Song X, Xie L, Tan X, Wang Z, Yang Y, Yuan Y, et al. (2014) A Multi-Center, Cross-Sectional Study on the Burden of Infectious Keratitis in China. PLoS ONE 9(12): e113843. https://doi.org/10.1371/ journal.pone.0113843

10. Tewari A, Sood N, Mahenrda M, Mehta D. Epidemiological and microbiological profile of infective keratitis in Ahmadabad. Indian J Ophthalmol. 2012;60(4):267-272.

11. Qiu S, Zhao GQ, Lin J, et al. Natamycin in the treatment of fungal keratitis: a systematic review and Meta-analysis. Int J Ophthalmol. 2015;8(3):597-602.

12. Gupta N, Tandan R. Investigative modalities in infectious keratitis. Indian J Ophthalmol. 2008;56(3):209-213.

13. Taylor PR, Lean Jr.SM, Sun Y, Pearlman E. Aspergillus and Fusarium corneal infections are regulated by Th17 cells and IL-17 producing neutrophils. J Immunol. 2014;192(7):3319-3327.

14. Barathi MJ, Ramakrishnan R, Minakshi R, Mittal S, Shivkumar C. Srinivasan M. Microbiological diagnosis of infective keratitis: a comparative evaluation of direct microscopy and culture results. Br J Ophthalmol. 2006;90(10):1271-1276.

15. Titiyal JS, Negi S, Anand A, Tandon R, Sharma N, Vajpayee RB. Risk factors for perforation in microbial corneal ulcers in north India. Br J Ophthalmol. 2006;90(6):686-689.

16. Srinivasan M, Gonzales CA, George C, et al. Epidemiology and etiological diagnosis of corneal ulceration in Madurai, south India. Br J Ophthalmol. 1997;81(11):965-971.

17. Ong HS, Fung SSM, Macleod D, Dart JKG, Tuft SJ, Burton MJ. Altered patterns of fungal keratitis at a London ophthalmic referral hospital: An eight-year retrospective observational study. Am J Ophthalmol. 2016;168: 227-236.

18. Ansari Z, Miller D, Galor A. Current thoughts in fungal keratitis: diagnosis and treatment. Curr Fungal Infect Rep. 2013;7(3):209-218. 
19. Rajaram $R$, Bhat $P$, Vaidee $V$, et al. Topical $5 \%$ Natamycin with oral ketoconazole in filamentous fungal keratitis: A randomized controlled trial. Asia Pac J Ophthalmol. 2015;4(3):146-150.

20. Al-Mujaini A, Al-Kharusi N, Thakral A, Wali UK. Bacterial keratitis: perspective on epidemiology, clinico-pathogenesis, diagnosis and treatment. Sultan Qaboos Univ Med J. 2009;9(2):184-95. Epub 2009 Jun 30. PMID: 21509299; PMCID: PMC3074777.

21. Tahereh S, Kiumars ND, Tahmineh MH. Fungal keratitis in patients with corneal ulcer in Sari, Northern Iran. Arch Iran Med. 2006;9(3):222-227. 